\title{
Communicable Diseases Report, NSW, March and April 2008
}

\author{
Communicable Diseases Branch, \\ NSW Department of Health
}

For updated information, including data and facts on specific diseases, visit www.health.nsw.gov.au and click on Infectious Diseases. The communicable diseases site now uses browser-friendly html formats to improve accessibility and, as a result, has a new address http://www.health.nsw.gov.au/ publichealth/infectious/index.asp.

Tables 1 and 2 and Figure 1 show reports of communicable diseases received through to the end of April 2008 in NSW.

\section{Measles continues to circulate in NSW}

Three confirmed cases of measles were notified during March in the Sydney area. One case of measles was confirmed in a partially immunised female aged in her twenties who had recently returned from England. An unimmunised contact who received Normal Human Immunoglobulin (NHIG) on day 7 post-exposure subsequently developed symptoms of sore throat, cough, coryza, fever and rash, and was confirmed with measles. A male aged in his forties, who recently returned from travel to Japan, was also confirmed with measles in March.

During April, a further seven cases of measles were confirmed in young adults ranging in age from 16 to 28 years, bringing the total number of cases to 20 in NSW this year. One of these cases was an international student from an English language college in Sydney. Three cases were subsequently linked to this case: a household contact; a staff member from the college; and a student from the college, all aged in their $20 \mathrm{~s}$.

In response to these cases, public health units across Sydney have conducted clinics to promote immunisation for susceptible contacts. Children and young adults born during or since 1966 who have never had measles and people who travel overseas should make sure they have had two doses of MMR vaccine. For more information, see: http://www.health.nsw.gov.au/PublicHealth/ Infectious/a-z.asp.

\section{Enterics disease}

In March, NSW public health units investigated 14 outbreaks of gastroenteritis including 10 suspected to be caused by person-to-person spread and four suspected to be foodborne.

Of the suspected person-to-person outbreaks, six were reported from aged care facilities where 81 people were affected. Four outbreaks were reported from childcare centres where 27 people were affected. All were suspected to be caused by viral infections, although norovirus was confirmed in only one outbreak that was in an aged care facility.

The four outbreaks that were suspected to be foodborne, affected 101 people (ranging from seven to 50 people per outbreak). In the largest outbreak, 50 of approximately 100 people residing in an institutional setting were ill with symptoms, including vomiting and diarrhoea. Clostridium perfringens toxin was identified in stool specimens from three ill patients. Epidemiological evidence suggested that a curry meal was the likely vehicle for infection. In another outbreak, Salmonella bacteria were identified in some of the stools of 14 people who were ill after eating a common meal. The epidemiological investigation suggested that a dessert that had included raw eggs was the likely vehicle. The cause of the remaining two outbreaks remains unclear.

In April, NSW public health units investigated 20 outbreaks of gastroenteritis including 16 where person-toperson spread was implicated, two suspected to be foodborne and two suspected to be related to an environmental exposure. The NSW Food Authority inspected commercial premises associated with these outbreaks.

The 16 outbreaks where person-to-person spread was suspected affected a total of 178 people. Eight occurred in child care centres and affected 82 people, seven occurred in aged care facilities and affected 90 people, and one occurred in a hospital and affected six people. Clinical specimens were submitted for testing from six of 13 suspected person-to-person gastroenteritis outbreaks. Rotavirus was confirmed in stool samples from one aged care facility outbreak, and in another both rotavirus and Norovirus were identified in stool samples. The causative agent was not confirmed for the remaining outbreaks.

Of the two suspected foodborne gastroenteritis outbreaks, one was a small cluster of three cases of Salmonella 
Typhimurium infection. All cases reported eating takeaway salad that contained mayonnaise dressing made from raw egg (a known risk factor for salmonellosis).

One of the suspected environmental exposure outbreaks was due to Shiga toxigenic Escherichia coli (STEC) O26 among a group of 250 Japanese students who were visiting Sydney during part of the incubation period for their illness. In total, 75 students (including 39 asymptomatic students) tested positive for STEC O26 after they returned home to Japan. STEC is carried by animals, such as cattle. People are infected when they come into contact with the faeces of an infected animal or person, either directly or indirectly. STEC is spread through consuming contaminated food (e.g. undercooked burgers, unwashed salad vegetables and unpasteurised milk or milk products), drinking or swimming in contaminated water, person-toperson contact (e.g. contact with faeces of an infected person) and contact with animals on farms or petting zoos. The students had visited a wildlife park and eaten at several restaurants. Despite an investigation, the source of infection, whether in Australia or Japan, remains unclear.

An outbreak of Salmonella Bioser Java that is clustered around the Northern Beaches area, and suspected to be due to an environmental exposure, is currently under investigation.

An outbreak of Salmonella Typhimurium (MLVA type 312-9-10-550) that was reported in February has continued throughout March and April. A total of 65 cases have now been reported with most infections occurring in March. Of the 65 cases, 37 (57\%) were male and the median age of cases was 19 years (range 1-84 years). Cases mainly lived in metropolitan Sydney and an exploratory investigation commenced in mid April. Hypothesis-generating interviews have been conducted and, although the source of the outbreak remains unclear, 13 of 18 cases reported eating eggs during the incubation period. Of these 13, seven reported eating raw eggs, including two young males who drank raw egg milkshakes. The NSW Food Authority is assisting with the ongoing investigation.

\section{Murray Valley Encephalitis}

In February 2008, Murray Valley Encephalitis (MVE) was detected in Culex annulorostris mosquitoes that were trapped near Griffith. In March 2008, MVE was detected in sentinel chicken flocks at Macquarie Marshes in western NSW and Leeton in the Riverina area of southern NSW. Seroconversions of sentinel chickens were also subsequently reported in three Victorian locations along the Murray River.

The majority of people infected with MVE will have no symptoms. Of those who do, symptoms include:

- high fever

- severe headache

- seizures or fits (especially in young children)

- tremors

- neck stiffness

- lethargy, irritability, drowsiness

- vomiting

- nausea

- diarrhoea

- dizziness

- confusion

- coma in severe cases.

Previous seroconversions occurred in flocks of sentinel chickens in NSW in 2001 and 2003 without associated human illness.

As part of enhanced surveillance for possible human cases of MVE, public health units from the Greater West, Hunter New England and Greater Southern Area Health Services have worked with selected local general practitioners to promote serological testing of patients presenting to general practitioners and local hospitals with consistent symptoms. No evidence of recent seroconversion to MVE has been found in those who were tested and there have been no reports of clinical cases of MVE in these areas to the end of April. As mosquito activity falls with the low temperatures in autumn and winter, the risk of human transmission is expected to decrease.

Public health units issued alerts to their local communities about avoiding mosquito bites. The advice included that people who live in or who visit these areas should avoid being outside in the late afternoon and at dusk, wear lightcoloured, long-sleeved, loose-fitting clothing and use an effective insect repellent. Residents should also remove any containers that may hold water from around their homes and fit fly screens to their windows and doors. 
Figure 1. Reports of selected communicable diseases, NSW, January 2004 to April 2008, by month of onset.

Preliminary data: case counts in recent months may increase because of reporting delays.

Laboratory-confirmed cases only, except for measles, meningococcal disease and pertussis.

BFV, Barmah Forest virus infections; RRV, Ross River virus infections; Lab Conf, laboratory confirmed;

Men $\mathrm{Gp} C$ and $\mathrm{Gp} B$, meningococcal disease due to serogroup $C$ and serogroup B infection;

other/unk, other or unknown serogroups.

NB: multiple series in graphs are stacked, except gastroenteritis outbreaks.

NB: Outbreaks are more likely to be reported by nursing homes and hospitals than by other institutions.

\begin{tabular}{|lc|}
\hline \multicolumn{2}{|c|}{ NSW Population } \\
Male & $50 \%$ \\
$<5$ y & $7 \%$ \\
$5-24$ y & $27 \%$ \\
$25-64$ y & $53 \%$ \\
$65+$ y & $13 \%$ \\
Rural & $46 \%$ \\
\hline
\end{tabular}
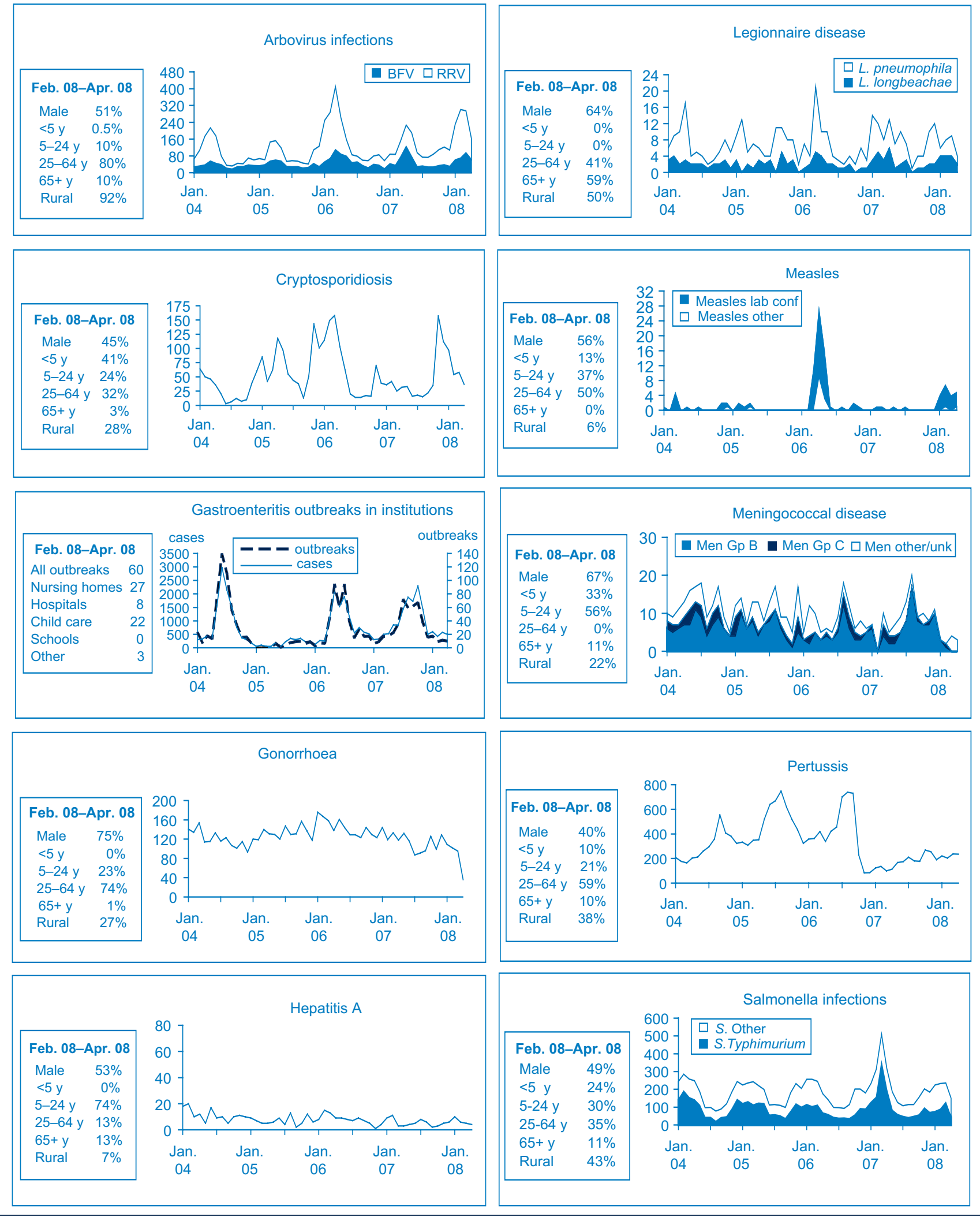
Table 1. Reports of notifiable conditions received in March 2008 by Area Health Services

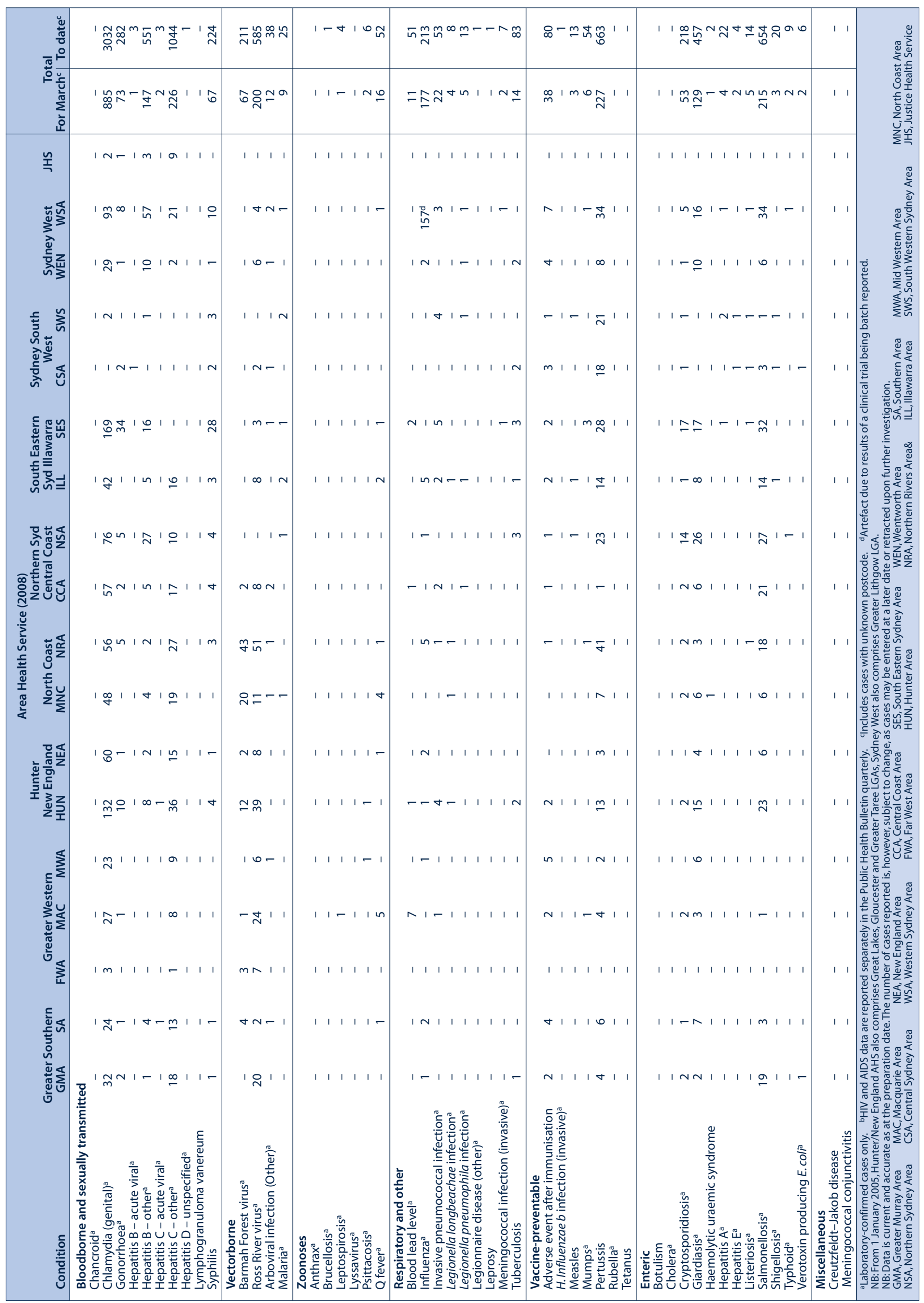


Table 2. Reports of notifiable conditions received in April 2008 by Area Health Services

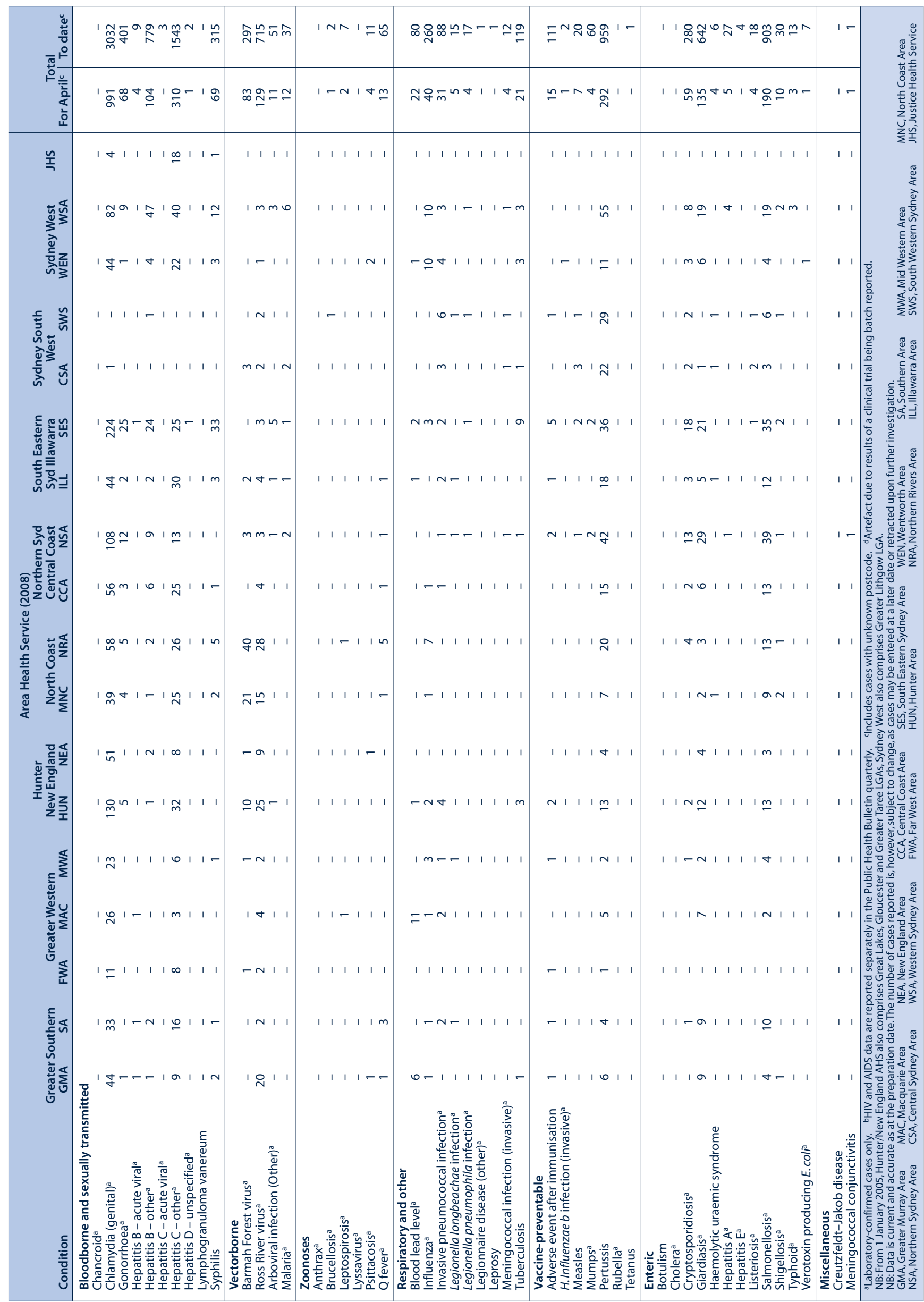

